\title{
Simulation of the Aerodynamic Behaviour of a Micro Wind Turbine
}

\author{
J. M. M. Monteiro ${ }^{1}$, J. C. Páscoa ${ }^{1}$ and F. M R. P. Brójo ${ }^{2}$ \\ ${ }^{1}$ Department of Electromechanical Engineering \\ ${ }^{2}$ Department of Aerospace Sciences and Technology \\ University of Beira Interior \\ R. Marquês D'Avila e Bolama - 6201-001 Covilhã (Portugal) \\ Fax number:+00351 275 329762, e-mail: monteiro@ubi.pt, pascoa@ubi.pt, brojo@ubi.pt
}

\begin{abstract}
.
In this paper the Computational Fluid Dynamics (CFD) simulation of a wind turbine with a rotor diameter of 2.2 meters is performed using the Fluent commercial code. Renewable energies are only effective if implemented in a large scale basis. Given this fact, this CFD study is part of the effort that is currently being made at the University of Beira Interior, Portugal, in order to develop a family of complete micro wind systems suitable for partial production by the final users. The target objectives of the project are to provide a quality, open source, small wind turbine project for the masses, with emphasis on low cost, reliability and ease construction. In a first phase battery charging systems are being developed, to be followed later by grid connected ones. The micro turbines being developed follow the mainstream configuration for this size class, and so are of the horizontal axis type, have three blades, a tip speed ratio of 6, constant pitch and a passive wind orientation system with side furling. The generators are of the axial flux permanent magnet type. The blades have a NACA 4415 wing section in all their span which is, at present, judged as a good compromise between performance and structural ruggedness given that it is a relatively thick profile. A 3D CAD model of the rotor was modelled with the Rhinoceros 3D program. In order to support the physical development of the wind turbine CFD simulations were performed. In this work results will be presented for turbulent flow computations around the wind turbine. The flow was computed by solving the Navier-Stokes equations with $k-\varepsilon$ turbulence model. A detailed analysis of the pressure distribution for three sections along the blade span is also presented. Load distribution and boundary layer separation is studied. Finally, a series of computations are performed in order to obtain the wind turbine torque and efficiency signature as a function of rotation. The obtained results will be, in the near future, compared to fullscale field tests. These will be carried out on a mobile test rig currently under development at University of Beira Interior, Portugal.
\end{abstract}

\section{Key words}

wind turbines, turbulence, CFD, low-Reynolds number

\section{Introduction}

Given the current energy crisis and global warming concerns, the last decade saw a considerable interest in the development of wind turbines, the main research efforts been targeted to grid connected, large units, with rated powers of the order of many thousands of watts.

In the present research, the focus is in the emerging micro-turbine arena, whose growth in importance can be attributed to government supporting policies stimulating the implementation of distributed sources of renewable energies and also to the recent trend of auto-construction of micro turbines by interested individuals [1].

The computational simulation of the aerodynamic behaviour of micro wind turbines is a particularly difficult task, mainly due to the low Reynolds number effects that were not correctly modelled by the previous generation of CFD codes. The usual option would be to test the physical geometry on wind tunnel or field tests. Fortunately there has been a remarkable improvement on CFD capabilities in recent years [2]. These are now able to compute 3D turbulent flow and rotational effects and can provide information on aerodynamic force and moment coefficients [3]. Presently, the main limitations are related to transition modelling. Reasonable results have been obtained using wall functions in conjunction with $k-\varepsilon$ turbulence model and other more elaborate methods based on the Boussinesque hypothesis [4]. According to several authors, the problem of using wall functions does not severely affect the results, as long as the flow remains attached. These assumptions can provide good results on normal forces and their associated bending moments. Nevertheless, care should be taken regarding the modelling of chord wise forces that, as usual, suffer from a lower confidence level.

Another CFD modelling problem is related to the effects of transition modelling and grid sensitivity at the tip sections of the blade [5-10]. Performing a fully turbulent modelling, without transition, has its consequences. Among these are an increased computed skin friction drag at all sections, a reduced numerical magnitude of the 
chord-wise force, and a reduced computed power, particularly at lower wind speeds. As the wind speed increases, the power generation is dominated by the normal component of the aerodynamic force, and to a smaller extent by the pressure drag component. Skin friction drag plays a progressive minor role. These drawbacks should be taken into account when doing an analysis of the results obtained in the present work. Nevertheless, the presented results are important in order to have an insight on wind turbine flow conditions in these early phases of the design stage.

\section{Micro Wind Turbine}

The blade shape was draw in the Rhinoceros 3D CAD program according to the formulas suggested by Piggott [1]. Blade sections at several stations from the wing tip were cut on a CNC mill machine. These sections had holes to allow a precise positioning along a steel spar, as can be seen in Fig. 1.

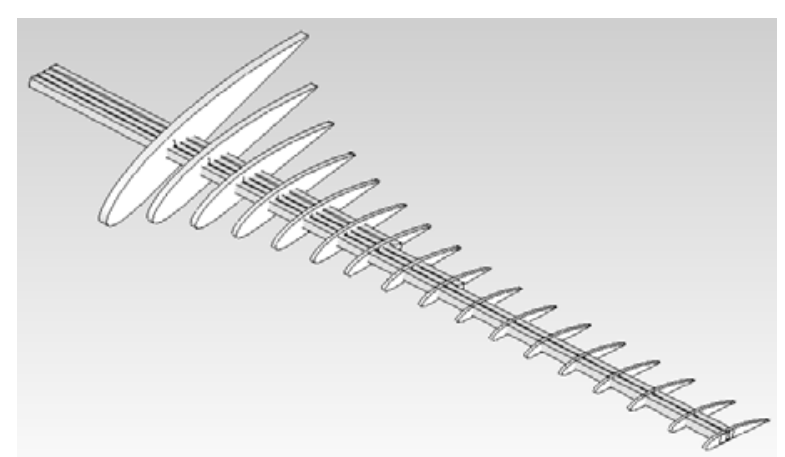

Fig. 1. 3D modelling of the internal structure of the wind turbine blade.

The space between the plywood sections was filled with foam that was shaped to allow a smooth transition between sections. The blades were coated with carbon fibber. In Fig. 2 an image of the constructed wind turbine is presented. It must be stressed out that the above described construction method will not be the definitive one, but just a quick way to obtain a prototype rotor for preliminary field tests.

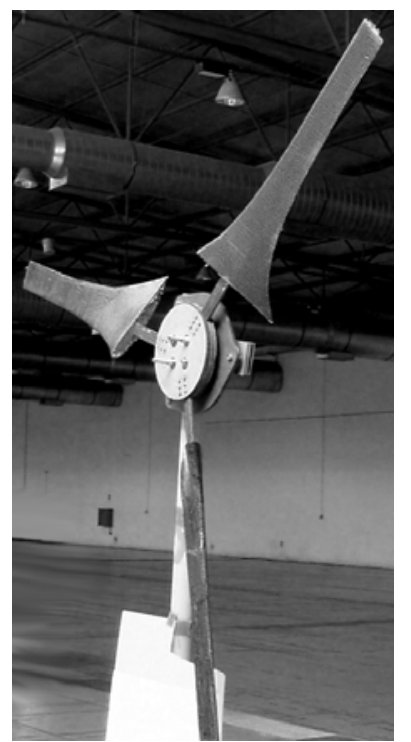

Fig. 2. Prototype of the micro wind turbine.

\section{Numerical Modelling}

The numerical model is based on the Fluent commercial code. Herein we solve the Navier-Stokes equations with the $k-\varepsilon$ turbulence model in 3D.

The geometry of the micro wind turbine, described in section 2, was discretized using an unstructured mesh. Figure 3 presents the mesh boundaries for one of the three wind turbine blades, actually the one used for the computations. The mesh comprises 311000 nodes, and extends 2 blade chords upwind and 4 blade chords downwind, as suggested in [4].

\section{A. Implementation of the Computational Model}

The MRF (Moving Reference Frame) option was used to incorporate the blade rotation. The code solves the Navier-Stokes equations with the $k-\varepsilon$ turbulence model and incorporates standard wall functions to compute the near wall flow. Second order discretization schemes for all the variables and the SIMPLE algorithm were selected to solve the pressure-velocity coupling. Albeit unsteady flow can take place, triggered by large scale eddies forming in the flow, the problem was solved for steady flow using an implicit discretization scheme. This is the standard design practice for this level of detail in the design chain.

The boundary conditions were implemented by selecting a velocity inlet upwind from the blades. An outflow

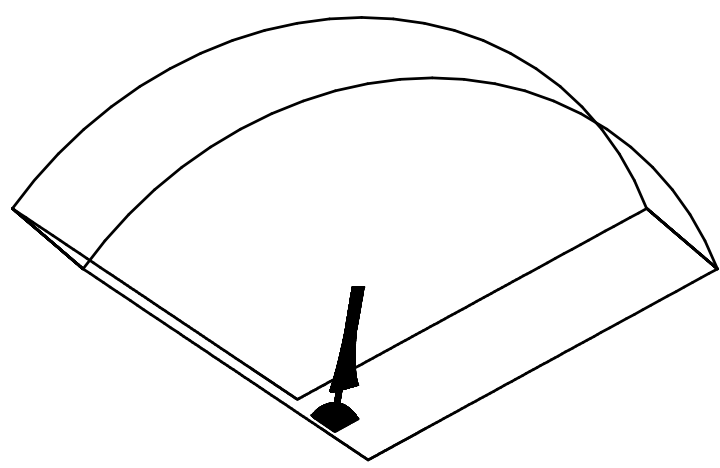

Fig. 3. Numerical domain used to discretize the wind turbine.

condition was specified downwind, by assuming that a fully developed flow occurs. For the top boundary several implementation strategies can be found in the literature. Besides the outflow boundary condition, herein implemented, some authors implemented a more sophisticated condition by incorporating an auxiliary row of cells, where the variables remain constant and their values are considered equal to the undisturbed flow. At inlet, turbulence intensity can usually change from 7-15\% 
at $10 \mathrm{~m} / \mathrm{s}$ as a function of the on site flow conditions. For all the computations performed in this work, a turbulence intensity of $10 \%$ was retained. The considered nominal wind turbine conditions were $10 \mathrm{~m} / \mathrm{s}$, for the wind absolute velocity, and $50 \mathrm{rad} / \mathrm{sec}$ for the rotation.

\section{B. Numerical Results}

The first computations were performed for the nominal working conditions mentioned above. Figure 4 presents the static pressure distribution around the blade surface. The coloured pressure distribution highlights the fact that the blade is uniformly loaded on the last 2/3 of the blade. At the tip, there is a strong $3 \mathrm{D}$ vortex structure that unloads the blade, but its effect is limited to a small portion of the blade tip. This is in accordance with the pressure center that is located at $0.61 \mathrm{~m}$, from the rotation axis, for this blade having an axis-tip length of $1.1 \mathrm{~m}$.

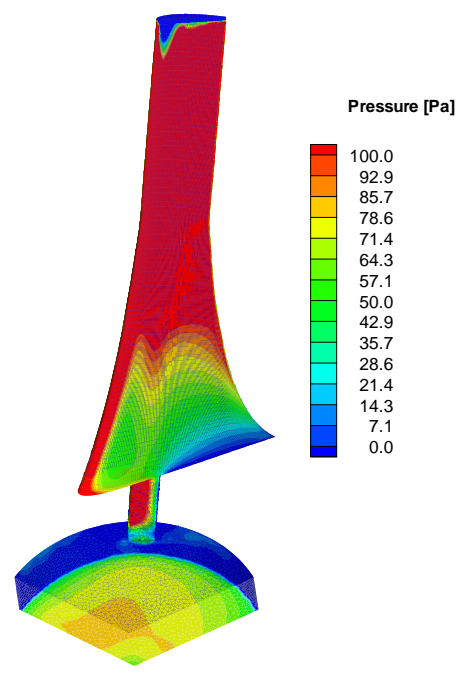

a)

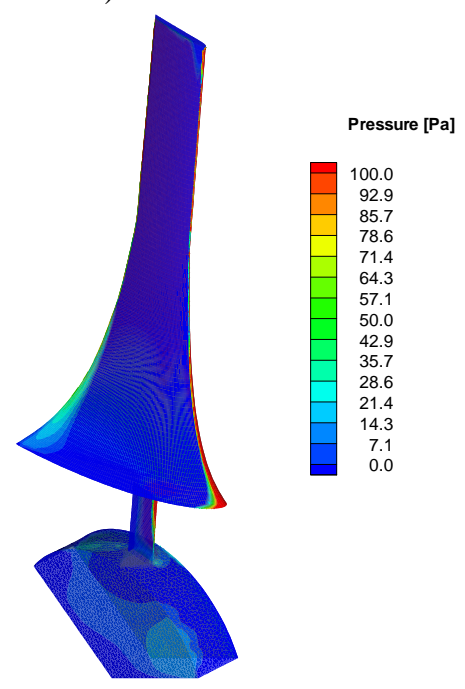

b)

Fig. 4. Results obtained for the static pressure, in SI units, at $10 \mathrm{~m} / \mathrm{s}$ inflow conditions: a) as seen from upwind; b) as seen from backwind.
In Fig. 5 can be seen the variation in blade load at 3 sections. The figure presents the blade pressure distribution as a function of non-dimensional axial chord. For the near hub section ( $0.3 \mathrm{~m}$ from rotating axis) the blade is almost completely loaded, except at the trailing edge where separation occurs. The same is not true for the near tip section (at $1.07 \mathrm{~m}$ ) were can be observed a massive separation starting from mid chord to the trailing edge. For the section at midspan (at $0.8 \mathrm{~m}$ ) can be seen that separation starts much later (around $86 \%$ chord as indicated by the arrow). Furthermore, in this section the pressure difference is quite high when compared with the other sections.

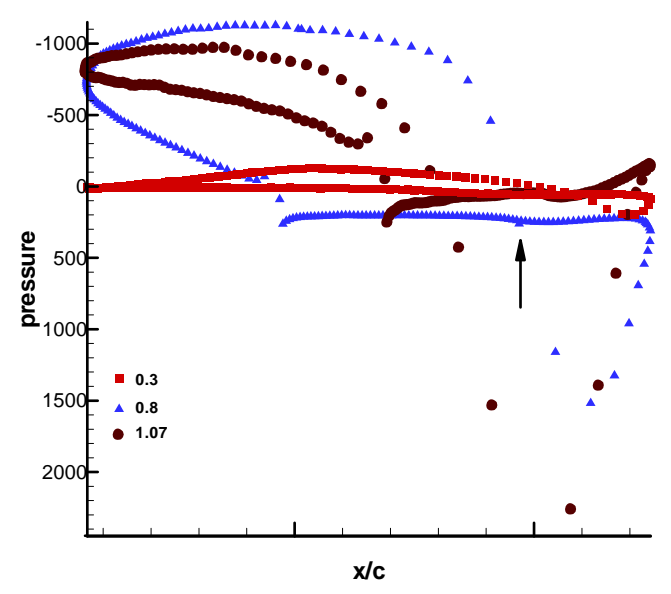

Fig. 5. Pressure distribution, in SI units, around a blade section of the wind turbine. Results are presented for a section near hub ( $0.3 \mathrm{~m}$ from rotating axis), for a section at midspan $(0.8 \mathrm{~m})$ and for a near tip section $(1.07 \mathrm{~m})$

Two more virtual tests were performed for incoming flow velocities of 5 and $2.5 \mathrm{~m} / \mathrm{s}$, in order to obtain the optimum load of the wind turbine for given inlet flow velocities. In Tab. 1 the results obtained for the torque, as a function of rotating speed, are presented for the three incoming flow velocities studied: 10,5 and $2.5 \mathrm{~m} / \mathrm{s}$. Highlighted is also the starting torque at $0 \mathrm{rpm}$, for $10 \mathrm{~m} / \mathrm{s}$ incoming velocity.

All computations were performed at the Almourol, Beowulf architecture computer cluster, of the Center for Aerospace Sciences and Technologies at UBI. This is a state-of-the-art high performance computing facility consisting of 6 heterogeneous CPUs. The exceptional performance of this system can be judged by the following example; for the present computations, the computational speed is 22.3 times faster than if performed on a Pentium 4 computer at $3 \mathrm{GHz}$. Albeit this, performing this kind of computations, for this complex real geometries, is not an easy task. Convergence was difficult to achieve, mostly due to the intricate geometry in the near hub region. This introduces complexities in the generation of the mesh, that where mitigated by resorting to an unstructured mesh. Minimum computing time for a virtual experimental point was 6 hours. 
Table I: Torque variation with angular velocity for the three studied incoming flow velocities: 10,5 and $2.5 \mathrm{~m} / \mathrm{s}$.

\begin{tabular}{|c|c|c|}
\hline $\begin{array}{c}\text { Incoming flow } \\
\text { velocity (m/s) }\end{array}$ & $\begin{array}{c}\text { Angular } \\
\text { velocity (rad/s) }\end{array}$ & $\begin{array}{c}\text { My } \\
\text { (N.m) }\end{array}$ \\
\hline 10 & 50 & 3.06 \\
\hline 10 & 40 & 4.43 \\
\hline 10 & 30 & 4.71 \\
\hline 10 & 20 & 4.51 \\
\hline 10 & 10 & 2.14 \\
\hline 10 & 0 & 1.50 \\
\hline 5 & 27 & 0.61 \\
\hline 5 & 17 & 1.11 \\
\hline 5 & 7 & 0.73 \\
\hline 2.5 & 14 & 0.12 \\
\hline 2.5 & 9 & 0.26 \\
\hline 2.5 & 4 & 0.28 \\
\hline 2.5 & 1.8 & 0.22 \\
\hline
\end{tabular}

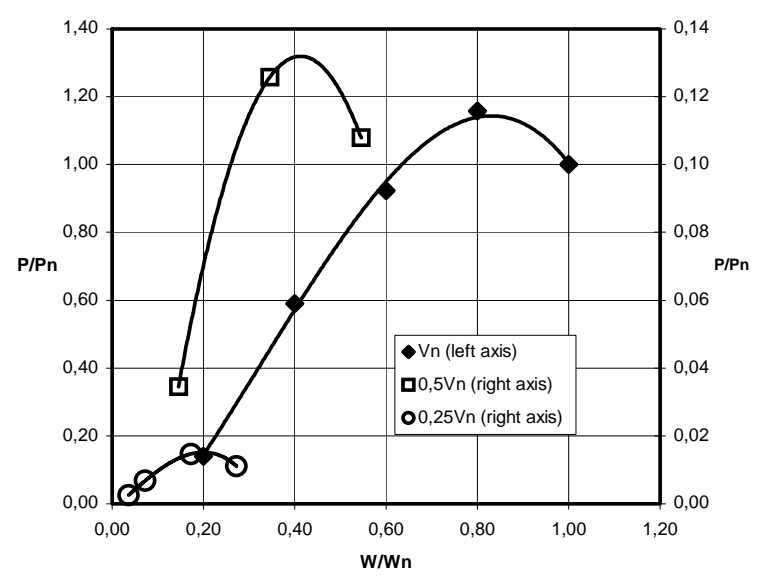

Fig. 6. Non-dimensional evolution of shaft power as a function of rotational speed for the three incoming velocities studied.

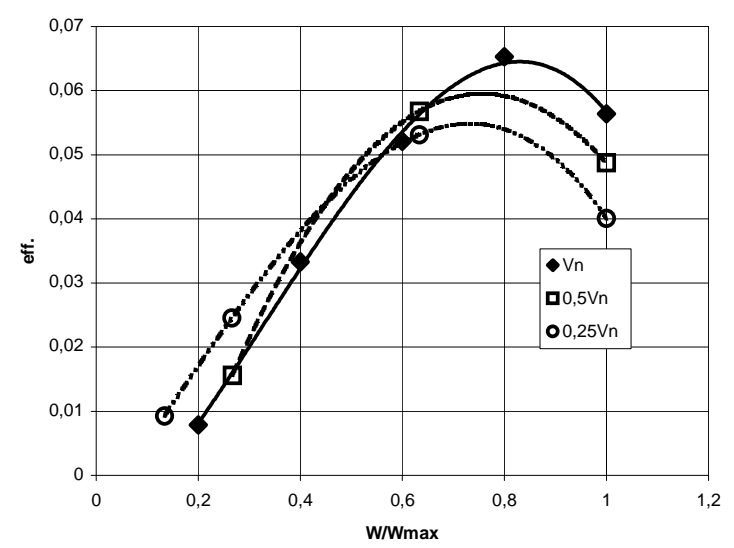

Fig. 7. Rotor efficiency as a function of angular velocity, non-dimensionalized by each local nominal rotational speeds.
The values of load, torque and moment calculated for all cases studied fluctuate somewhat with time. This is especially the case for the computer runs performed with an incoming speed of $10 \mathrm{~m} / \mathrm{s}$, due to periodic separation and vortex shedding phenomena. For the present computations pure steady-state flow conditions were assumed.

Figure 6 presents the evolution of non-dimensional shaft power as a function of non-dimensional rotational speed, for the three incoming flow velocities. The rotational speed used in this non-dimensionalization was $50 \mathrm{rad} / \mathrm{s}$, which corresponds to an incoming flow velocity of $10 \mathrm{~m} / \mathrm{s}$ and a design tip speed ratio of 6 . Shaft power was non-dimensionalized by the power obtained at $50 \mathrm{rad} / \mathrm{s}$ and $10 \mathrm{~m} / \mathrm{s}$ inflow velocity. Due to the great disparity of values, a double axis was used for the vertical scale. It can be seen that the power attains a maximum for each of the curves corresponding to different inlet conditions. Despite the limited number of points, it is clear that computations captured the overall behaviour of the machine, by obtaining points above and below the maximum power in all three cases. It can be concluded that the maximum power extraction is obtained with a load imposed by the electrical generator that translates into an $80 \%$ reduction in the design angular velocity, for a tip speed ratio of 6 . This data is extremely valuable for the implementation of the peak-power tracker methodology [11], a system that is currently been developed for the electrical control of the wind turbine. In Fig. 7 is depicted the rotor's efficiency as a function of the angular velocity, non-dimensionalized by local maximum angular velocity. This local maximum angular velocity is considered for a tip-speed ratio of 6 for each of the incoming flow speeds. Once again, it can be observed that there is an optimal rotational speed, herein obtained for the maximum efficiency. The maximum efficiency is obtained for the highest incoming flow velocity of $10 \mathrm{~m} / \mathrm{s}$. Low Reynolds number effects, causing separation of the flow, are a cause of the reduced values obtained for the efficiency at the lower incoming velocities.

\section{Conclusion}

This work presents one component of the research that is being carried out at Universidade da Beira Interior in order to design micro wind turbines. In this paper we focused on the numerical studies performed for the wind turbine prototype built at UBI. The extensive numerical tests performed allowed the knowledge of some important aerodynamic characteristics of the wind turbine.

The results herein presented will help refine the design of the electric project of the wind turbine, in particular its control system.

Future numerical studies will focus two main subjects. First, the incorporation of a spinner and the evaluation of its presence on the turbine's efficiency. Second, to implement a detailed study of non-steady aerodynamic effects. This will be carried out by running the code simulations for unsteady flow. 
The results obtained in this study will be in the near future compared with full-scale tests on an experimental set-up currently under development.

\section{Acknowledgements}

The authors would like to thank Mr. João Correia for the assistance in manufacturing of the prototype. Financial support was also provided by CAST - Center for Aerospace Sciences and Technology at UBI.

\section{References}

[1] H. Piggott, "Windpower Workshop" The Centre for Alternative Technology, ISBN 978-1-898049-27-2

[2] C. Masson and A. Smaïli, "Numerical study of turbulent flow around a wind nacelle", Wind Energy, Vol. 9, pp. 281-298, 2008.

[3] C. A. Baxevanou, P. K. Chaviaropoulos, S. G. Voutsinas and N. S. Vlachos, "Evaluations study of a Navier-Stokes CFD aeroelastic model of wind turbine airfoils in classical flutter”, Jounal of Wind Engineering and Industrial Aerodynamics, Vol. 96, pp. 1425-1443, 2008.

[4] S. Benjanirat and L. Sankar, "Evaluation of turbulence models for the prediction of wind turbine aerodynamics”, AIAA paper 0517, 2003.

[5] E. Ferrer and X. Munduate, "Wind turbine blade tip comparison using CFD”, Journal of Physics: Conference Series, Vol. 75, 2007.

[6] K. W. Ayotte, "Computational modelling for wind energy assessment”, Journal of Wind Engineering and Industrial Aerodynamics, Vol. 96, pp. 1571-1590, 2008.

[7] C. Tongchitpakdee, S. Benjanirat and L. N. Sankar, "Numerical simulation of the aerodynamics of horizontal axis wind turbines under yawed flow conditions", Journal of solar energy engineering, Vol. 127, $\mathrm{N}^{\circ} 4$, pp. 156, 2005.

[8] S. Frandsen, H. E. Jorgensen and J. D. Sorensen, "Relevant criteria for testing the quality of turbulence models”, Journal of solar energy engineering, Vol. 130, No 3, pp. 1016, 2008.

[9] C. H. J. Stork, C. P. Butterfield, W. Holley, P. H. Madsen and P. H. Jensen, "Wind conditions for wind turbine design proposals for revision of the IEC 1400-1 standard”, Journal of Wind Engineering and Industrial Aerodynamics, Vol. 74-76, pp. 443-454, 1998.

[10] A. Kasmin and C. Masson, “An extended k-e model for turbulent flow through horizontal-axis wind turbines”, Journal of Wind Engineering and Industrial Aerodynamics, Vol. 96, pp. 103-122, 2008.

[11] A. M. De Broe, S. Drouilhet and V. Gevorgian, “A peak power tracker for small wind turbines in battery charging applications” Energy conversion, ieee transactions on, Vol. 14, No 4, pp. 1630-1635, 1999. 